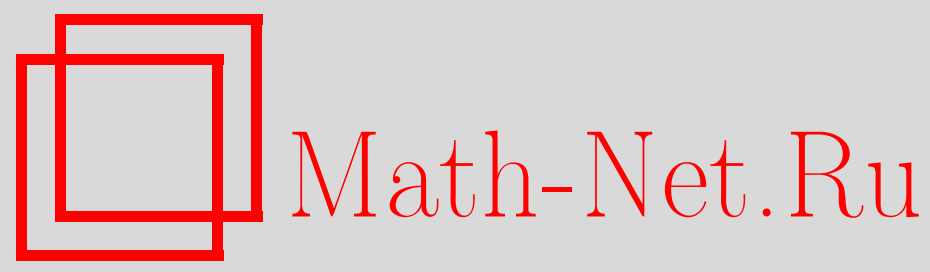

О. М. Джохадзе, Влияние младших членов на корректность постановки характеристических задач для гиперболических уравнений третьего порядка, Матем. заметки, 2003, том 74, выпуск 4, 517-528

DOI: https://doi.org/10.4213/mzm282

Использование Общероссийского математического портала Math-Net.Ru подразумевает, что вы прочитали и согласны с пользовательским соглашением http://www.mathnet.ru/rus/agreement

Параметры загрузки:

IP : 54.224 .60 .19

26 апреля 2023 г., 13:18:58

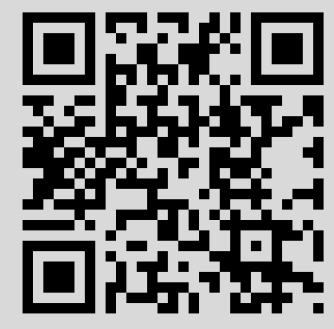




\section{ВЛИЯНИЕ МЛАДШИХ ЧЛЕНОВ НА КОРРЕКТНОСТЬ ПОСТАНОВКИ ХАРАКТЕРИСТИЧЕСКИХ ЗАДАЧ ДЛЯ ГИПЕРБОЛИЧЕСКИХ УРАВНЕНИЙ ТРЕТЬЕГО ПОРЯДКА}

\section{о.М. Джохадзе}

В работе для гиперболического уравнения третьего порядка общего вида с доминированными младшими членами рассмотрена общая задача типа Гурса. Выявлены эффекты влияния младших членов, присутствующих как в уравнении, так и в граничных условиях, на корректность поставленной задачи.

Библиографоия: 16 названий.

В плоскости независимых переменных $x, y$ рассмотрим гиперболическое уравнение третьего порядка обшего вида с доминированньми (см., например, [1]) младшими членами

$$
L u:=u_{x x y}+a^{2,0} u_{x x}+a^{1,1} u_{x y}+a^{1,0} u_{x}+a^{0,1} u_{y}+a^{0,0} u=f,
$$

где $a^{i, j}, i=0,1,2, j=0,1, i+j \neq 3, f$-заданные, а $u$-искомая действительные функции.

Прямые $y=$ const образуют двукратное семейство характеристик уравнения (1), а прямые $x=$ const - однократное.

Пусть

$$
\gamma_{1}: y=0, \quad 0 \leqslant x<\infty, \quad \gamma_{2}: x=0, \quad 0 \leqslant y<\infty,
$$

- характеристические лучи уравнения (1). Обозначим через $D$ область, ограниченную лучами $\gamma_{1}, \gamma_{2}$ и расположенную в угле $x>0, y>0$.

Пусть $P_{1}^{0}$ и $P_{2}^{0}$ - точки пересечения $\gamma_{1}$ и $\gamma_{2}$ соответственно с характеристиками $L_{1}\left(P^{0}\right): x=x_{0}$ и $L_{2}\left(P^{0}\right): y=y_{0}$, выходящими из произвольно взятой точки $P^{0}\left(x_{0}, y_{0}\right) \in D$. Уравнение (1) будем рассматривать в прямоугольной области

$$
D_{0}:=\left\{(x, y): 0<x<x_{0}, 0<y<y_{0}\right\}
$$

ограниченной характеристиками $x=0, x=x_{0}$ и $y=0, y=y_{0}$.

Для уравнения (1) рассмотрим общую характеристическую задачу типа Гурса в следующей постановке: требуется найти в $D_{0}$ регулярное решение $u$ уравнения $(1)$, удовлетворяюшее на отрезках $O P_{1}^{0}$ и $O P_{2}^{0}$ лучей $\gamma_{1}$ и $\gamma_{2}$ следуюшим граничным условиям:

$$
\begin{aligned}
& \left.\left(M_{1} u_{x x}+N_{1} u_{x y}+P_{1} u_{x}+Q_{1} u_{y}+S_{1} u\right)\right|_{O P_{1}^{0}}=f_{1}, \\
& \left.\left(M_{2} u_{x x}+N_{2} u_{x y}+P_{2} u_{x}+Q_{2} u_{y}+S_{2} u\right)\right|_{O P_{2}^{0}}=f_{2}, \\
& \left.\left(M_{3} u_{x x}+N_{3} u_{x y}+P_{3} u_{x}+Q_{3} u_{y}+S_{3} u\right)\right|_{O P_{2}^{0}}=f_{3},
\end{aligned}
$$

где $M_{i}, N_{i}, P_{i}, Q_{i}, S_{i}, f_{i}, i=1,2,3,-$ заданные действительные функции. 
Регулярным решением уравнения (1) называется функция $u$, непрерьвная в $D_{0}$ вместе со своими частными производньми $D_{x}^{i} D_{y}^{j} u, i=0,1,2, j=0,1, i+j>0, D_{x}:=\partial / \partial x$, $D_{y}:=\partial / \partial y$, и удовлетворяющая уравнению $(1)$ в $D_{0}$.

ЗАмЕчАНИЕ 1. Отметим, что гиперболическая природа рассматриваемой задачи учтена наличием в ней лишь производных, доминированных главной частью $D_{x}^{2} D_{y} u$ уравнения (1).

ЗАмЕчАнИЕ 2. Ввиду того, что семейство характеристик $y=$ const является двукратньм для гиперболического уравнения (1), на отрезке $O P_{2}^{0}$ луча $\gamma_{2}$ заданы два условия (3), (4).

Отметим, что гиперболические уравнения третьего и более высокого порядка с доминированными младшими членами, которые иногда называют псевдопараболическими, встречаются при изучении вопросов фильтрации жидкости в трещиноватых средах, влагопереноса в почвогрунтах, передачи тепла в гетерогенных средах, моделировании различных биологических процессов и явлений, продольного колебания в тонком упругом стержне с учетом эффектов поперечной инерции, распространения волн в диспергирующих средах, а также в теории оптимальных процессов и обратных задач и т. д. (см., например, [2]-[7]).

Одно из семейств характеристик уравнения (1) является кратным, и этот фактор существенно влияет как на корректность постановки задач, так и на характер их разрешимости.

Введем в рассмотрение следующие функциональные пространства:

$$
\begin{aligned}
C_{0}\left(\bar{D}_{0}\right) & :=\left\{u: u \in C\left(\bar{D}_{0}\right), u(O)=0\right\}, \quad O:=(0,0), \\
C_{0}[0, d] & :=\{\varphi: \varphi \in C[0, d], \varphi(0)=0\}, \\
C^{m, n}\left(\bar{D}_{0}\right) & :=\left\{u: D_{x}^{i} u, D_{y}^{j} u \in C\left(\bar{D}_{0}\right), 0 \leqslant i \leqslant m, 0 \leqslant j \leqslant n\right\}, \\
C^{0,0}\left(\bar{D}_{0}\right) & :=C\left(\bar{D}_{0}\right), \quad C^{m, 0}\left(\bar{D}_{0}\right):=C^{m}\left(\bar{D}_{0}\right), \quad C^{0, n}\left(\bar{D}_{0}\right):=C^{n}\left(\bar{D}_{0}\right), \\
C_{0}^{m, n}\left(\bar{D}_{0}\right) & :=\left\{u: D_{x}^{i} u, D_{y}^{j} u \in C_{0}\left(\bar{D}_{0}\right), i=0,1, \ldots, m, j=0,1, \ldots, n\right\}, \\
C_{0}^{m}[0, d] & :=\left\{\varphi: D_{t}^{i} \varphi \in C_{0}[0, d], i=0,1, \ldots, m\right\}, \quad m=0,1, \ldots, \quad n=0,1, \ldots, d>0 .
\end{aligned}
$$

Граничную задачу (1)-(4) будем исследовать в пространстве $C_{0}^{2,1}\left(\bar{D}_{0}\right)$ и в этом случае будем требовать, чтобы

$$
\begin{gathered}
a^{i, j} \in C^{i, j}\left(\bar{D}_{0}\right), \quad i=0,1,2, \quad j=0,1, \quad i+j \neq 3, \\
f \in C_{0}\left(\bar{D}_{0}\right), \quad M_{1}, N_{1}, P_{1}, Q_{1}, S_{1} \in C\left[0, x_{0}\right], \\
M_{i}, N_{i}, P_{i}, Q_{i}, S_{i} \in C\left[0, y_{0}\right], \quad i=2,3, \quad f_{1} \in C_{0}\left[0, x_{0}\right], \quad f_{i} \in C_{0}\left[0, y_{0}\right], \quad i=2,3 .
\end{gathered}
$$

Пусть вьполнены следующие условия:

$$
M_{1}(x) \neq 0, \quad 0 \leqslant x \leqslant x_{0},
$$

И

$$
\Delta(y):=\operatorname{det}\left\|\begin{array}{ll}
Q_{2}(y) & N_{2}(y) \\
Q_{3}(y) & N_{3}(y)
\end{array}\right\| \neq 0, \quad 0 \leqslant y \leqslant y_{0} .
$$

В работах автора (см., например, [8]-[11]) доказана следующая 
ТЕОрема 1. Пусть выполнены условия (5), (6). Тогда задача (1)-(4) однозначно разрешима в классе $C_{0}^{2,1}\left(\bar{D}_{0}\right)$.

Естественно возникает вопрос: что происходит, когда достаточные условия (5) и (6) разрешимости задачи (1)-(4) отдельно или одновременно нарушаются на отрезках $O P_{1}^{0}$ и $O P_{2}^{0}$ соответственно. При нарушении условий (5) или (6), как показывает простой пример уравнения $u_{x x y}=0$, задача $(1)-(4)$ может оказаться некорректнопоставленной. Ниже будет показано, что наличие младших членов в уравнении (1) и в граничных условиях (2)-(4) может оказать влияние на корректность постановки задачи (1)-(4) (см., например, [12]-[14]).

1. Согласно предшествуюшим работам (см., например, [9], [10], [15], [16]), функция Римана $v(x, y ; \xi, \eta),(x, y ; \xi, \eta) \in \bar{D}_{0} \times \bar{D}_{0}$, уравнения (1) однозначно определяется как решение задачи Гурса

$$
\begin{gathered}
L_{(x, y)}^{*} v:=-v_{x y x}+\left(a^{2,0} v\right)_{x x}+\left(a^{1,1} v\right)_{x y}-\left(a^{1,0} v\right)_{x}-\left(a^{0,1} v\right)_{y}+a^{0,0} v=0 \\
v(\xi, y ; \xi, \eta)=0, \quad v_{x}(\xi, y ; \xi, \eta)=\exp \left\{\int_{\eta}^{y} a^{2,0}\left(\xi, y_{1}\right) d y_{1}\right\} \\
v(x, \eta ; \xi, \eta)=\omega_{0}(x, \eta ; \xi, \eta)
\end{gathered}
$$

где $(\xi, \eta)$ - произвольная фиксированная точка из замкнутой области $\bar{D}_{0}$. Здесь $\omega_{0}(x, \eta ; \xi, \eta)$ - решение линейного обыкновенного дифференциального уравнения второго порядка

$$
v_{x x}(x, \eta ; \xi, \eta)-\left(a^{1,1} v\right)_{x}(x, \eta ; \xi, \eta)+\left(a^{0,1} v\right)(x, \eta ; \xi, \eta)=0
$$

относительно переменной $x$, удовлетворяющее следующим начальным условиям Коши:

$$
\left.v(x, \eta ; \xi, \eta)\right|_{x=\xi}=0,\left.\quad v_{x}(x, \eta ; \xi, \eta)\right|_{x=\xi}=1
$$

В то же время известно, что (см., например, [15])

$$
D_{x}^{i} D_{y}^{j} v, D_{\xi}^{i} D_{\eta}^{j} v \in C\left(\bar{D}_{0} \times \bar{D}_{0}\right), \quad i=0,1,2, \quad j=0,1,
$$

и для регулярного решения $u$ уравнения (1) имеет место следующее интегральное представление:

$$
\begin{aligned}
u(x, y)= & \int_{0}^{x}\left[v_{x}(\xi, 0 ; x, y) \varphi_{1}^{\prime}(\xi)+\left(a^{1,1} v\right)_{x}(\xi, 0 ; x, y) \varphi_{1}(\xi)-\left(a^{0,1} v\right)(\xi, 0 ; x, y) \varphi_{1}(\xi)\right] d \xi \\
& -\int_{0}^{y}\left[v(0, \eta ; x, y) \nu_{1}^{\prime}(\eta)+\left(a^{2,0} v\right)(0, \eta ; x, y) \nu_{1}(\eta)\right] d \eta \\
& -\int_{0}^{y}\left[v_{x y}(0, \eta ; x, y)-\left(a^{2,0} v\right)_{x}(0, \eta ; x, y)-\left(a^{1,1} v\right)_{y}(0, \eta ; x, y)\right. \\
& \left.+\left(a^{1,0} v\right)(0, \eta ; x, y)\right] \psi_{1}(\eta) d \eta-\left(a^{1,1} v\right)(0, y ; x, y) \psi_{1}(y)+v_{x}(0, y ; x, y) \psi_{1}(y) \\
& +\left(a^{1,1} v\right)(0,0 ; x, y) \varphi_{1}(0)-\int_{0}^{x} \int_{0}^{y} v(\xi, \eta ; x, y) f(\xi, \eta) d \xi d \eta
\end{aligned}
$$

где $\varphi_{1}(x):=u(x, 0), 0 \leqslant x \leqslant x_{0}, \psi_{1}(y):=u(0, y), \nu_{1}(y):=u_{x}(0, y), 0 \leqslant y \leqslant y_{0}$. 
2. Пусть условие (5) нарушено на всем отрезке $O P_{1}^{0}: y=0,0 \leqslant x \leqslant x_{0}$, т.е.

$$
M_{1}(x)=0, \quad 0 \leqslant x \leqslant x_{0},
$$

а условие (6) вьполнено.

В этом случае для простоты изложения, будем считать, что $N_{1}, P_{1}, Q_{1}, S_{1}=$ const, $f_{1} \in C_{0}^{1}\left[0, x_{0}\right]$ и $M_{i}, N_{i}, P_{i}, Q_{i}, S_{i} \in C\left[0, y_{0}\right], f_{i} \in C_{0}\left[0, y_{0}\right], i=2,3$.

Случай $i) N_{1} \neq 0$. В этом случае граничное условие (2) можно рассматривать как обыкновенное дифференциальное уравнение первого порядка относительно функции $\varphi_{*}(x):=u_{y}(x, 0), 0 \leqslant x \leqslant x_{0}, \varphi_{*} \in C_{0}^{2}\left[0, x_{0}\right]$,

$$
\varphi_{*}^{\prime}(x)+N_{1}^{-1} Q_{1} \varphi_{*}(x)=N_{1}^{-1}\left[f_{1}(x)-P_{1} \varphi_{1}^{\prime}(x)-S_{1} \varphi_{1}(x)\right], \quad 0 \leqslant x \leqslant x_{0},
$$

где $\varphi_{1} \in C_{0}^{2}\left[0, x_{0}\right]$.

Решение уравнения $(9)$ класса $C_{0}^{2}\left[0, x_{0}\right]$ имеет следующий вид:

$$
\begin{aligned}
\varphi_{*}(x)= & -N_{1}^{-1} P_{1} \varphi_{1}(x)-N_{1}^{-1}\left(S_{1}-N_{1}^{-1} P_{1} Q_{1}\right) \int_{0}^{x} \exp \left\{N_{1}^{-1} Q_{1}(\xi-x)\right\} \varphi_{1}(\xi) d \xi \\
& +N_{1}^{-1} \int_{0}^{x} \exp \left\{N_{1}^{-1} Q_{1}(\xi-x)\right\} f_{1}(\xi) d \xi, \quad 0 \leqslant x \leqslant x_{0} .
\end{aligned}
$$

Рассматривая уравнение $(1)$ на отрезке $0 \leqslant x \leqslant x_{0}$ характеристической прямой $y=0$ и учитывая (10), относительно неизвестной функции $\varphi_{1} \in C_{0}^{2}\left[0, x_{0}\right]$ получаем следующее интегро-дифференциальное уравнение второго порядка:

$$
\begin{aligned}
{\left[P_{1}-\right.} & \left.N_{1} a^{2,0}(x, 0)\right] \varphi_{1}^{\prime \prime}(x)+\left[P_{1} a^{1,1}(x, 0)-N_{1} a^{1,0}(x, 0)+S_{1}-N_{1}^{-1} P_{1} Q_{1}\right] \varphi_{1}^{\prime}(x) \\
+ & {\left[\left(S_{1}-N_{1}^{-1} P_{1} Q_{1}\right) a^{1,1}(x, 0)+P_{1} a^{0,1}(x, 0)\right.} \\
& \left.-N_{1} a^{0,0}(x, 0)-N_{1}^{-1} Q_{1}\left(S_{1}-N_{1}^{-1} P_{1} Q_{1}\right)\right] \varphi_{1}(x) \\
+ & {\left[-N_{1}^{-1} Q_{1} a^{1,1}(x, 0)+a^{0,1}(x, 0)+N_{1}^{-2} Q_{1}^{2}\right]\left(S_{1}-N_{1}^{-1} P_{1} Q_{1}\right) } \\
& \times \int_{0}^{x} \exp \left\{N_{1}^{-1} Q_{1}(\xi-x)\right\} \varphi_{1}(\xi) d \xi=\tilde{f}(x), \quad 0 \leqslant x \leqslant x_{0}
\end{aligned}
$$

где

$$
\begin{aligned}
\tilde{f}(x):= & f_{1}^{\prime}(x)+\left[a^{1,1}(x, 0)-N_{1}^{-1} Q_{1}\right] f_{1}(x) \\
+ & {\left[-N_{1}^{-1} Q_{1} a^{1,1}(x, 0)+a^{0,1}(x, 0)+N_{1}^{-2} Q_{1}^{2}\right] } \\
& \times \int_{0}^{x} \exp \left\{N_{1}^{-1} Q_{1}(\xi-x)\right\} f_{1}(\xi) d \xi-N_{1}^{-1} f(x, 0), \quad 0 \leqslant x \leqslant x_{0} .
\end{aligned}
$$

Для получения интегро-дифференциальных соотношений относительно неизвестных функций $\psi_{1}, \nu_{1}$ поступим следующим образом. Рассматривая уравнение (1) на отрезке $0 \leqslant y \leqslant y_{0}$ характеристической прямой $x=0$ относительно функции $\psi_{*}(y):=u_{x x}(0, y)$, $0 \leqslant y \leqslant y_{0}, \psi_{*} \in C_{0}^{1}\left[0, y_{0}\right]$, получаем следующее обыкновенное дифференциальное уравнение первого порядка:

$$
\begin{aligned}
\psi_{*}^{\prime}(y)+a^{2,0}(0, y) \psi_{*}(y)= & f(0, y)-a^{1,1}(0, y) \nu_{1}^{\prime}(y)-a^{1,0}(0, y) \nu_{1}(y) \\
& -a^{0,1}(0, y) \psi_{1}^{\prime}(y)-a^{0,0}(0, y) \psi_{1}(y), \quad 0 \leqslant y \leqslant y_{0},
\end{aligned}
$$


решение которого представляется по формуле

$$
\begin{aligned}
\psi_{*}(y)=\int_{0}^{y}\left[f(0, \eta)-a^{1,1}(0, \eta) \nu_{1}^{\prime}(\eta)-a^{1,0}(0, \eta) \nu_{1}(\eta)-a^{0,1}(0, \eta) \psi_{1}^{\prime}(\eta)\right. \\
\left.-a^{0,0}(0, \eta) \psi_{1}(\eta)\right] \exp \left\{\int_{y}^{\eta} a^{2,0}(0, t) d t\right\} d \eta, \quad 0 \leqslant y \leqslant y_{0} .
\end{aligned}
$$

Подставляя $\psi_{*}$ из (12) в граничные условия (3), (4), получаем следующую систему интегро-дифференциальных уравнений первого порядка относительно неизвестных функций $\psi_{1}, \nu_{1} \in C_{0}^{1}\left[0, y_{0}\right]$ :

$$
\begin{aligned}
& N_{i} \nu_{1}^{\prime}(y)+Q_{i} \psi_{1}^{\prime}(y)+P_{i} \nu_{1}(y)+S_{i} \psi_{1}(y) \\
& -M_{i} \int_{0}^{y}\left[a^{1,1}(0, \eta) \nu_{1}^{\prime}(\eta)+a^{1,0}(0, \eta) \nu_{1}(\eta)+a^{0,1}(0, \eta) \psi_{1}^{\prime}(\eta)+a^{0,0}(0, \eta) \psi_{1}(\eta)\right] \\
& \quad \times \exp \left\{\int_{y}^{\eta} a^{2,0}(0, t) d t\right\} d \eta \\
& =f_{i}(y)-M_{i} \int_{0}^{y} f(0, \eta) \exp \left\{\int_{y}^{\eta} a^{2,0}(0, t) d t\right\} d \eta, \quad 0 \leqslant y \leqslant y_{0}, \quad i=2,3 .
\end{aligned}
$$

ЗАмЕчАНИЕ 3. Очевидно, что при вьполнении условий (6), (8) задача (1)-(4) в классе $C_{0}^{2,1}\left(\bar{D}_{0}\right)$ эквивалентна системе уравнений $(11),(13)$ относительно неизвестных $\varphi_{1} \in$ $C_{0}^{2}\left[0, x_{0}\right], \psi_{1}, \nu_{1} \in C_{0}^{1}\left[0, y_{0}\right]$

Хорошо известно, что система уравнений (13) при выполнении условия (6) однозначно разрешима относительно функций $\psi_{1}, \nu_{1} \in C_{0}^{1}\left[0, y_{0}\right]$. Поэтому вопрос разрешимости задачи (1)-(4) в классе $C_{0}^{2,1}\left(\bar{D}_{0}\right)$ при выполнении условий $(6),(8)$ эквивалентным образом редуцируется к вопросу разрешимости уравнения (11) относительно $\varphi_{1} \in C_{0}^{2}\left[0, x_{0}\right]$.

Пусть вьполнено условие

$$
P_{1}-N_{1} a^{2,0}(x, 0) \neq 0, \quad 0 \leqslant x \leqslant x_{0},
$$

тог да уравнение (11) однозначно разрешимо в классе $C_{0}^{2}\left[0, x_{0}\right]$.

Пусть теперь условие (14) нарушено всюду, т.е.

$$
P_{1}-N_{1} a^{2,0}(x, 0)=0, \quad 0 \leqslant x \leqslant x_{0} .
$$

В этом случае уравнение (11) принимает вид

$$
\begin{aligned}
& {\left[P_{1} a^{1,1}(x, 0)-N_{1} a^{1,0}(x, 0)+S_{1}-N_{1}^{-1} P_{1} Q_{1}\right] \varphi_{1}^{\prime}(x)} \\
& +\left[\left(S_{1}-N_{1}^{-1} P_{1} Q_{1}\right) a^{1,1}(x, 0)+P_{1} a^{0,1}(x, 0)\right. \\
& \left.\quad-N_{1} a^{0,0}(x, 0)-N_{1}^{-1} Q_{1}\left(S_{1}-N_{1}^{-1} P_{1} Q_{1}\right)\right] \varphi_{1}(x) \\
& +\left[-N_{1}^{-1} Q_{1} a^{1,1}(x, 0)+a^{0,1}(x, 0)+N_{1}^{-2} Q_{1}^{2}\right]\left(S_{1}-N_{1}^{-1} P_{1} Q_{1}\right) \\
& \quad \times \int_{0}^{x} \exp \left\{N_{1}^{-1} Q_{1}(\xi-x)\right\} \varphi_{1}(\xi) d \xi=\tilde{f}(x), \quad 0 \leqslant x \leqslant x_{0} .
\end{aligned}
$$

При выполнении условия

$$
P_{1} a^{1,1}(x, 0)-N_{1} a^{1,0}(x, 0)+S_{1}-N_{1}^{-1} P_{1} Q_{1} \neq 0, \quad 0 \leqslant x \leqslant x_{0},
$$


для однозначной разрешимости уравнения $(16)$ в классе $C_{0}^{2}\left[0, x_{0}\right]$ следует дополнительно потребовать, чтобы

$$
a^{0,1}(\cdot, 0), a^{0,0}(\cdot, 0), f(\cdot, 0), f_{1}^{\prime} \in C^{1}\left[0, x_{0}\right], \quad f_{1}^{\prime \prime}(0)-N_{1}^{-1} f_{x}(0,0)=0 .
$$

Теперь рассмотрим случай, когда условие (17) нарушено на всем отрезке $y=0$, $0 \leqslant x \leqslant x_{0}$, т.е.

$$
P_{1} a^{1,1}(x, 0)-N_{1} a^{1,0}(x, 0)+S_{1}-N_{1}^{-1} P_{1} Q_{1}=0, \quad 0 \leqslant x \leqslant x_{0} .
$$

Учитывая (19), уравнение (16) перепишем в виде

$$
\begin{aligned}
{\left[\left(S_{1}-\right.\right.} & \left.N_{1}^{-1} P_{1} Q_{1}\right) a^{1,1}(x, 0)+P_{1} a^{0,1}(x, 0)-N_{1} a^{0,0}(x, 0) \\
& \left.-N_{1}^{-1} Q_{1}\left(S_{1}-N_{1}^{-1} P_{1} Q_{1}\right)\right] \varphi_{1}(x) \\
+ & \left.-N_{1}^{-1} Q_{1} a^{1,1}(x, 0)+a^{0,1}(x, 0)+N_{1}^{-2} Q_{1}^{2}\right]\left(S_{1}-N_{1}^{-1} P_{1} Q_{1}\right) \\
& \times \int_{0}^{x} \exp \left\{N_{1}^{-1} Q_{1}(\xi-x)\right\} \varphi_{1}(\xi) d \xi=\widetilde{f}(x), \quad 0 \leqslant x \leqslant x_{0} .
\end{aligned}
$$

Пусть теперь вьполнено условие

$$
\begin{gathered}
\left(S_{1}-N_{1}^{-1} P_{1} Q_{1}\right) a^{1,1}(x, 0)+P_{1} a^{0,1}(x, 0)-N_{1} a^{0,0}(x, 0)-N_{1}^{-1} Q_{1}\left(S_{1}-N_{1}^{-1} P_{1} Q_{1}\right) \neq 0, \\
0 \leqslant x \leqslant x_{0} .
\end{gathered}
$$

Тогда для однозначной разрешимости уравнения (20) относительно $\varphi_{1} \in C_{0}^{2}\left[0, x_{0}\right]$ наряду с условиями (18) следует дополнительно потребовать, чтобы

$$
\begin{gathered}
a^{1,1}(\cdot, 0), a^{0,1}(\cdot, 0), a^{0,0}(\cdot, 0), f(\cdot, 0), f_{1}^{\prime} \in C^{2}\left[0, x_{0}\right], \\
f_{1}^{\prime \prime \prime}(0)+\left[a^{1,1}(0,0)-N_{1}^{-1} Q_{1}\right] f_{1}^{\prime \prime}(0)-N_{1}^{-1} f_{x x}(0,0)=0 .
\end{gathered}
$$

В этом случае для получения явного решения уравнения (20) введем обозначение

$$
\varphi_{2}(x):=\int_{0}^{x} \exp \left\{N_{1}^{-1} Q_{1} \xi\right\} \varphi_{1}(\xi) d \xi, \quad 0 \leqslant x \leqslant x_{0} .
$$

Очевидно, что относительно $\varphi_{2}$ с учетом (20) имеем уравнение

$$
\varphi_{2}^{\prime}(x)+\widetilde{b}(x) \varphi_{2}(x)=\exp \left\{N_{1}^{-1} Q_{1} x\right\} \widetilde{a}(x) \tilde{f}(x), \quad 0 \leqslant x \leqslant x_{0}
$$

где $\widetilde{a}$ и $\widetilde{b}$ - вполне определенные функции.

Решая последнее уравнение, с учетом условия Коши $\varphi_{2}(0)=0$ окончательно находим $\varphi_{1}(x)=\widetilde{a}(x) \widetilde{f}(x)-\widetilde{b}(x) \int_{0}^{x} \exp \left\{N_{1}^{-1} Q_{1}(\xi-x)+\int_{x}^{\xi} \widetilde{b}(t) d t\right\} \widetilde{a}(\xi) \widetilde{f}(\xi) d \xi, \quad 0 \leqslant x \leqslant x_{0}$.

Рассмотрим теперь случай, когда условие (21) нарушено всюду на $O P_{1}^{0}$, т.е.

$$
\begin{gathered}
\left(S_{1}-N_{1}^{-1} P_{1} Q_{1}\right) a^{1,1}(x, 0)+P_{1} a^{0,1}(x, 0)-N_{1} a^{0,0}(x, 0)-N_{1}^{-1} Q_{1}\left(S_{1}-N_{1}^{-1} P_{1} Q_{1}\right)=0, \\
0 \leqslant x \leqslant x_{0} .
\end{gathered}
$$


В этом случае уравнение (20) принимает вид

$$
\begin{aligned}
& {\left[-N_{1}^{-1} Q_{1} a^{1,1}(x, 0)+a^{0,1}(x, 0)+N_{1}^{-2} Q_{1}^{2}\right]\left(S_{1}-N_{1}^{-1} P_{1} Q_{1}\right)} \\
& \quad \times \int_{0}^{x} \exp \left\{N_{1}^{-1} Q_{1}(\xi-x)\right\} \varphi_{1}(\xi) d \xi=\widetilde{f}(x), \quad 0 \leqslant x \leqslant x_{0} .
\end{aligned}
$$

\section{При выполнении условия}

$$
\left[-N_{1}^{-1} Q_{1} a^{1,1}(x, 0)+a^{0,1}(x, 0)+N_{1}^{-2} Q_{1}^{2}\right]\left(S_{1}-N_{1}^{-1} P_{1} Q_{1}\right) \neq 0, \quad 0 \leqslant x \leqslant x_{0},
$$

для однозначной разрешимости уравнения (24) относительно $\varphi_{1} \in C_{0}^{2}\left[0, x_{0}\right]$ наряду с условиями (18) и (22), приведенными вьше, следует дополнительно потребовать, чтобы

$$
\begin{gathered}
a^{1,1}(\cdot, 0), a^{0,1}(\cdot, 0), f(\cdot, 0), f_{1}^{\prime} \in C^{3}\left[0, x_{0}\right] \\
f_{1}^{\prime \prime \prime \prime}(0)+\left[3 a_{x}^{1,1}(0,0)-N_{1}^{-1} Q_{1} a^{1,1}(0,0)+a^{0,1}(0,0)+N_{1}^{-2} Q_{1}^{2}\right] f_{1}^{\prime \prime}(0) \\
+\left[a^{1,1}(0,0)-N_{1}^{-1} Q_{1}\right] f_{1}^{\prime \prime \prime}(0)-N_{1}^{-1} f_{x x x}(0,0)=0 .
\end{gathered}
$$

Решение уравнения (24), как и вьше, в этом случае представляется в явном виде

$$
\varphi_{1}(x)=N_{1}^{-1} Q_{1} M(x)+M^{\prime}(x), \quad 0 \leqslant x \leqslant x_{0},
$$

где

$$
\begin{gathered}
M(x):=\left\{\left[-N_{1}^{-1} Q_{1} a^{1,1}(x, 0)+a^{0,1}(x, 0)+N_{1}^{-2} Q_{1}^{2}\right]\left(S_{1}-N_{1}^{-1} P_{1} Q_{1}\right)\right\}^{-1} \tilde{f}(x), \\
0 \leqslant x \leqslant x_{0} .
\end{gathered}
$$

Пусть теперь условие (25) нарушено всюду, т.е.

$$
\left[-N_{1}^{-1} Q_{1} a^{1,1}(x, 0)+a^{0,1}(x, 0)+N_{1}^{-2} Q_{1}^{2}\right]\left(S_{1}-N_{1}^{-1} P_{1} Q_{1}\right)=0, \quad 0 \leqslant x \leqslant x_{0} .
$$

В этом случае левая часть уравнения (24) тождественно равна нулю и равенство

$$
\widetilde{f}(x)=0, \quad 0 \leqslant x \leqslant x_{0}
$$

является необходимым и достаточным условием для разрешимости уравнения (24) в классе $C_{0}^{2}\left[0, x_{0}\right]$, причем любая функция из этого класса является его решением.

В силу замечания 3 получаем, что справедлива следующая

ТЕОрема 2. ${ }^{1}$ Пусть выполнены условия (6), (8) и $N_{1} \neq 0$. Тогда при выполнении условия (14) задача (1)-(4) однозначно разрешима.

Если же имеет место (15), то при выполнении условия (17) задача (1)-(4) однозначно разрешима при дополнительном выполнении условий (18).

Далее, если выполнены условия (15) и (19), то при (21) задача (1)-(4) однозначно разрешима, если дополнительно выполнены условия (18) и (22).

\footnotetext{
${ }^{1}$ Всюду в сформулированных в этом пункте теоремах вопрос разрешимости задачи (1)-(4) рассматривается в классе $C_{0}^{2,1}\left(\bar{D}_{0}\right)$.
} 
В случае, когда имеют место равенства (15), (19), (23) и выполнено условие (25), то задача (1)-(4) однозначно разрешима, если дополнительно выполнень условия (18), (22) и (26).

Наконеи, пусть выполнены условия (15), (19), (23) и (27). Тогда для разрешимости задачи (1)-(4) необходимо и достаточно, чтобы выполнялось тохсдество (28), причем соответствующая (1)-(4) однородная задача имеет бесконечное множество линейно независимых решений, которые согласно (7) даются формулой

$$
\begin{gathered}
u_{0}(x, y)=\int_{0}^{x}\left[v_{x}(\xi, 0 ; x, y) \varphi_{1}^{\prime}(\xi)+\left(a^{1,1} v\right)_{x}(\xi, 0 ; x, y) \varphi_{1}(\xi)-\left(a^{0,1} v\right)(\xi, 0 ; x, y) \varphi_{1}(\xi)\right] d \xi \\
(x, y) \in \bar{D}_{0},
\end{gathered}
$$

әде $\varphi_{1}$ - произвольная функиия класса $C_{0}^{2}\left[0, x_{0}\right]$, a $v(x, y ; \xi, \eta)-$ функиия Римана уравнения (1).

Случай $i$ ) $N_{1}=0$. В этом случае граничное условие (2) принимает вид

$$
P_{1} u_{x}(x, 0)+Q_{1} u_{y}(x, 0)+S_{1} u(x, 0)=f_{1}(x), \quad 0 \leqslant x \leqslant x_{0} .
$$

Дифференцируя равенство (30) по переменной $x$, будем иметь

$$
P_{1} u_{x x}(x, 0)+Q_{1} u_{x y}(x, 0)+S_{1} u_{x}(x, 0)=f_{1}^{\prime}(x), \quad 0 \leqslant x \leqslant x_{0} .
$$

Очевидно, что равенства (30) и (31) являются эквивалентньми.

Рассматривая в качестве граничного условия (2) равенство (31), в силу теоремы 1 заключаем, что справедлива следующая

ТеОрема 3. Пусть выполнены условия (6), (8), $N_{1}=0 u P_{1} \neq 0$. Тогда задача (1)-(4) однозначно разрешима.

Случай $i$ ii) $N_{1}=P_{1}=0$. В этом случае условие (30) принимает вид

$$
Q_{1} \varphi_{*}(x)+S_{1} \varphi_{1}(x)=f_{1}(x), \quad 0 \leqslant x \leqslant x_{0} .
$$

Отсюда в предположении, что $Q_{1} \neq 0$, непосредственно следует

$$
\varphi_{*}(x)=Q_{1}^{-1}\left[f_{1}(x)-S_{1} \varphi_{1}(x)\right], \quad 0 \leqslant x \leqslant x_{0} .
$$

Рассматривая уравнение (1) на отрезке $O P_{1}^{0}$ при дополнительном требовании, что $f_{1} \in C_{0}^{2}\left[0, x_{0}\right]$, и подставляя в нем значение функции $\varphi_{*}$, представленной по формуле $(32)$, относительно функции $\varphi_{1} \in C_{0}^{2}\left[0, x_{0}\right]$ получаем обькновенное дифференциальное уравнение второго порядка следующего вида:

$$
\begin{aligned}
{\left[S_{1}-\right.} & \left.Q_{1} a^{2,0}(x, 0)\right] \varphi_{1}^{\prime \prime}(x)+\left[S_{1} a^{1,1}(x, 0)-Q_{1} a^{1,0}(x, 0)\right] \varphi_{1}^{\prime}(x) \\
& \quad+\left[S_{1} a^{0,1}(x, 0)-Q_{1} a^{0,0}(x, 0)\right] \varphi_{1}(x) \\
& =f_{1}^{\prime \prime}(x)+a^{1,1}(x, 0) f_{1}^{\prime}(x)+a^{0,1}(x, 0) f_{1}(x)-Q_{1} f(x, 0), \quad 0 \leqslant x \leqslant x_{0} .
\end{aligned}
$$

Очевидно, что при вьполнении условий (6), (8) и $N_{1}=P_{1}=0$ задача (1)-(4) в классе $C_{0}^{2,1}\left(\bar{D}_{0}\right)$ эквивалентна системе уравнений $(33)$ и (13) относительно $\varphi_{1} \in C_{0}^{2}\left[0, x_{0}\right]$ и $\psi_{1}, \nu_{1} \in C_{0}^{1}\left[0, y_{0}\right]$. 
Уравнение (33) исследуется так же, как и (11). Для простоты изложения обозначим через a), b) , . . условия, фигурируюшие при формулировании окончательного результата в виде теоремы:

a) $S_{1}-Q_{1} a^{2,0}(x, 0) \neq 0,0 \leqslant x \leqslant x_{0}$;

b) $S_{1}-Q_{1} a^{2,0}(x, 0)=0,0 \leqslant x \leqslant x_{0}$;

c) $S_{1} a^{1,1}(x, 0)-Q_{1} a^{1,0}(x, 0) \neq 0,0 \leqslant x \leqslant x_{0}$;

d) $a^{0,1}(\cdot, 0), a^{0,0}(\cdot, 0), f(\cdot, 0), f_{1}^{\prime \prime} \in C^{1}\left[0, x_{0}\right], f_{1}^{\prime \prime \prime}(0)-Q_{1} f_{x}(0,0)=0$;

e) $S_{1} a^{1,1}(x, 0)-Q_{1} a^{1,0}(x, 0)=0,0 \leqslant x \leqslant x_{0}$;

f) $S_{1} a^{0,1}(x, 0)-Q_{1} a^{0,0}(x, 0) \neq 0,0 \leqslant x \leqslant x_{0}$;

g) $a^{1,1}(\cdot, 0), a^{0,1}(\cdot, 0), a^{0,0}(\cdot, 0), f(\cdot, 0), f_{1}^{\prime \prime} \in C^{2}\left[0, x_{0}\right]$, $f_{1}^{\prime \prime \prime \prime}(0)+a^{1,1}(0,0) f_{1}^{\prime \prime \prime}(0)-Q_{1} f_{x x}(0,0)=0 ;$

h) $S_{1} a^{0,1}(x, 0)-Q_{1} a^{0,0}(x, 0)=0,0 \leqslant x \leqslant x_{0}$;

j) $f_{1}^{\prime \prime}(x)+a^{1,1}(x, 0) f_{1}^{\prime}(x)+a^{0,1}(x, 0) f_{1}(x)-Q_{1} f(x, 0)=0,0 \leqslant x \leqslant x_{0}$.

Теорема 4. Пусть выполнены условия (6), (8), $N_{1}=P_{1}=0 u Q_{1} \neq 0$.

Тогда при выполнении а) задача (1)-(4) однозначно разрешима.

Если же имеет место b), то при с) задача (1)-(4) однозначно разрешима при дополнительном выполнении $\mathrm{d})$.

Далее, в случае, когда имеют место b) и е), при f) задача (1)-(4) однозначно разрешима, если дополнительно выполнены d) $и \mathrm{~g})$.

Наконеи, пусть имеют место b), е) и h). Тогда для разрешимости задачи (1)-(4) необходимо и достаточно, чтобы выполнялось ј), причем соответствующая (1)-(4) однородная задача имеет бесконечное мнохество линейно независимых решений, которые в силу (7) даются формулой (29).

Случай $i v) N_{1}=P_{1}=Q_{1}=0$. Тогда естественно потребовать, чтобы $S_{1} \neq 0$, ибо в противном случае граничное условие (2), которое в данном случае принимает вид

$$
S_{1} u(x, 0)=f_{1}(x), \quad 0 \leqslant x \leqslant x_{0},
$$

не имело бы смысла.

Требуя дополнительно, чтобы $f_{1} \in C_{0}^{2}\left[0, x_{0}\right]$, и дифференцируя дважды равенство (34) по $x$, получаем

$$
S_{1} u_{x x}(x, 0)=f_{1}^{\prime \prime}(x), \quad 0 \leqslant x \leqslant x_{0} .
$$

Отметим, что равенства (34) и (35) являются эквивалентными.

Рассматривая в качестве граничного условия (2) равенство (35), в силу теоремы 1 заключаем, что справедлива следующая

ТЕОрема 5. При выполнении условий (6), (8), $N_{1}=P_{1}=Q_{1}=0$ и $S_{1} \neq 0$ задача (1)-(4) однозначно разрешима.

3. Пусть теперь условие (6) нарушено всюду на отрезке $O P_{2}^{0}: x=0,0 \leqslant y \leqslant y_{0}$, т.е.

$$
\Delta(y)=0, \quad 0 \leqslant y \leqslant y_{0}
$$

а условие (5) вьполнено.

В этом случае для простоты изложения будем считать, что $M_{i}, N_{i}, P_{i}, Q_{i}, S_{i}=$ const, $f_{i} \in C_{0}^{1}\left[0, y_{0}\right], i=2,3$, и $M_{1}, N_{1}, P_{1}, Q_{1}, S_{1} \in C\left[0, x_{0}\right], f_{1} \in C_{0}\left[0, x_{0}\right]$. 
Рассматривая уравнение (1) на характеристическом отрезке $O P_{1}^{0}: y=0,0 \leqslant x \leqslant x_{0}$, с учетом граничного условия (2) относительно вектора-столбца

$$
\Phi(x):=\left\|\begin{array}{c}
\varphi_{*}(x) \\
\varphi_{1}(x)
\end{array}\right\|, \quad 0 \leqslant x \leqslant x_{0},
$$

класса $C_{0}^{2}\left[0, x_{0}\right]$ получаем следующее обыкновенное дифференщиальное уравнение второго порядка вида

$$
A_{0}(x) \Phi^{\prime \prime}(x)+A_{1}(x) \Phi^{\prime}(x)+A_{2}(x) \Phi(x)=F(x), \quad 0 \leqslant x \leqslant x_{0} .
$$

Здесь

$$
A_{0}(x):=\left\|\begin{array}{cc}
1 & a^{2,0}(x, 0) \\
0 & M_{1}(x)
\end{array}\right\|, \quad 0 \leqslant x \leqslant x_{0},
$$

а $A_{1}, A_{2}$ - вполне определенные матрицы-функции, $F$ - известная вектор-функция.

Учитывая вид матрицы $A_{0}$, в силу условия (5) имеем

$$
\operatorname{det} A_{0}(x)=M_{1}(x) \neq 0, \quad 0 \leqslant x \leqslant x_{0} .
$$

Отсюда получаем, что система (37) однозначно разрешима в классе $C_{0}^{2}\left[0, x_{0}\right]$, следовательно, однозначно определяется и функция $\varphi_{1} \in C_{0}^{2}\left[0, x_{0}\right]$.

Далее, в силу (36) без ограничения общности будем считать, что справедливы следуюшие равенства

$$
N_{3}=M N_{2}, \quad Q_{3}=M Q_{2},
$$

где $M$ - вполне определенное действительное число.

С учетом этих равенств и граничных условий $(3),(4)$ относительно функций $\psi_{1}, \nu_{1} \in$ $C_{0}^{1}\left[0, y_{0}\right]$ на отрезке $O P_{2}^{0}$ получаем следующую систему функционально-дифференциальных уравнений:

$$
\begin{gathered}
N_{2} \nu_{1}^{\prime}(y)+Q_{2} \psi_{1}^{\prime}(y)+P_{2} \nu_{1}(y)+S_{2} \psi_{1}(y)=f_{2}(y)-\psi_{*}(y), \quad 0 \leqslant y \leqslant y_{0} \\
\left(M P_{2}-P_{3}\right) \nu_{1}(y)+\left(M S_{2}-S_{3}\right) \psi_{1}(y) \\
=M f_{2}(y)-f_{3}(y)-\left(M M_{2}-M_{3}\right) \psi_{*}(y), \quad 0 \leqslant y \leqslant y_{0} .
\end{gathered}
$$

Пусть выполнено условие

$$
M P_{2}-P_{3} \neq 0 \quad\left(M S_{2}-S_{3} \neq 0\right) .
$$

Определяя $\nu_{1}\left(\psi_{1}\right)$ из второго уравнения системы $(38)$ и подставляя ее в первое, при дополнительном условии

$$
Q_{2}\left(M P_{2}-P_{3}\right)-N_{2}\left(M S_{2}-S_{3}\right) \neq 0,
$$

находим, что единственное решение системы (38) имеет следующий вид:

$$
\begin{array}{ll}
\psi_{1}(y)=a_{1} \psi_{*}(y)+b_{1} \int_{0}^{y} \exp \{c(\eta-y)\} \psi_{*}(\eta) d \eta+F_{1}(y), & 0 \leqslant y \leqslant y_{0} \\
\nu_{1}(y)=a_{2} \psi_{*}(y)+b_{2} \int_{0}^{y} \exp \{c(\eta-y)\} \psi_{*}(\eta) d \eta+F_{2}(y), & 0 \leqslant y \leqslant y_{0}
\end{array}
$$

где $a_{i}, b_{i}, c$ и $F_{i}, i=1,2,-$ вполне определенные постоянные и функции соответственно, конкретные значения которьх для наших исследований принципиального значения не имеют. 
Теперь, рассматривая уравнение (1) на характеристическом отрезке $O P_{2}^{0}: x=0$, $0 \leqslant y \leqslant y_{0}$, и подставляя в нем значения функций $\psi_{1}$ и $\nu_{1}$ представленных по формулам (41), после простых преобразований получаем следующее интегро-дифференциальное уравнение первого порядка относительно $\psi_{*} \in C_{0}^{1}\left[0, y_{0}\right]$ :

$$
\begin{aligned}
\left\{1+a_{2} a^{1,1}(0, y)+a_{1} a^{0,1}(0, y)\right\} \psi_{*}^{\prime}(y) & \\
+ & \left\{a^{2,0}(0, y)+b_{2} a^{1,1}(0, y)+a_{2} a^{1,0}(0, y)+b_{1} a^{0,1}(0, y)+a_{1} a^{0,0}(0, y)\right\} \psi_{*}(y) \\
+ & \left\{-c b_{2} a^{1,1}(0, y)+b_{2} a^{1,0}(0, y)-c b_{1} a^{0,1}(0, y)+b_{1} a^{0,0}(0, y)\right\} \\
& \quad \times \int_{0}^{y} \exp \{c(\eta-y)\} \psi_{*}(\eta) d \eta \\
= & f(0, y)-a^{1,1}(0, y) F_{2}^{\prime}(y)-a^{1,0}(0, y) F_{2}(y) \\
& -a^{0,1}(0, y) F_{1}^{\prime}(y)-a^{0,0}(0, y) F_{1}(y), \quad 0 \leqslant y \leqslant y_{0} .
\end{aligned}
$$

ЗАмЕчАнИЕ 4. Очевидно, что при вьполнении условий (5), (36), (39), (40) задача (1)-(4) в классе $C_{0}^{2,1}\left(\bar{D}_{0}\right)$ эквивалентна системе интегро-дифференциальных уравнений (37), (42) относительно функций $\Phi \in C_{0}^{2}\left[0, x_{0}\right], \psi_{*} \in C_{0}^{1}\left[0, y_{0}\right]$.

Уравнение (42) исследуется так же, как и уравнения (11) и (33). Для простоты изложения, как и в п. 2 , обозначим через $\left.\left.\mathrm{a}^{\prime}\right), \mathrm{b}^{\prime}\right), \ldots$ условия, фигурирующие при формулировании окончательного результата в виде теоремы:

$$
\begin{aligned}
&\left.\mathrm{a}^{\prime}\right) 1+a_{2} a^{1,1}(0, y)+a_{1} a^{0,1}(0, y) \neq 0,0 \leqslant y \leqslant y_{0} ; \\
&\left.\mathrm{b}^{\prime}\right) 1+a_{2} a^{1,1}(0, y)+a_{1} a^{0,1}(0, y)=0,0 \leqslant y \leqslant y_{0} ; \\
&\left.\mathrm{c}^{\prime}\right) a^{2,0}(0, y)+b_{2} a^{1,1}(0, y)+a_{2} a^{1,0}(0, y)+b_{1} a^{0,1}(0, y)+a_{1} a^{0,0}(0, y) \neq 0,0 \leqslant y \leqslant y_{0} ; \\
&\left.\mathrm{d}^{\prime}\right) a^{1,0}(0, \cdot), a^{0,0}(0, \cdot), f(0, \cdot), F_{1}^{\prime}, F_{2}^{\prime} \in C^{1}\left[0, y_{0}\right] \\
& f_{y}(0,0)-a^{1,1}(0,0) F_{2}^{\prime \prime}(0)-a^{0,1}(0,0) F_{1}^{\prime \prime}(0)=0 ; \\
&\left.\mathrm{e}^{\prime}\right) a^{2,0}(0, y)+b_{2} a^{1,1}(0, y)+a_{2} a^{1,0}(0, y)+b_{1} a^{0,1}(0, y)+a_{1} a^{0,0}(0, y)=0,0 \leqslant y \leqslant y_{0} ; \\
&\left.\mathrm{f}^{\prime}\right)-c b_{2} a^{1,1}(0, y)+b_{2} a^{1,0}(0, y)-c b_{1} a^{0,1}(0, y)+b_{1} a^{0,0}(0, y) \neq 0,0 \leqslant y \leqslant y_{0} ; \\
&\left.\mathrm{g}^{\prime}\right) a^{1,1}(0, \cdot), a^{1,0}(0, \cdot), a^{0,1}(0, \cdot), a^{0,0}(0, \cdot), f(0, \cdot), F_{1}^{\prime}, F_{2}^{\prime} \in C^{2}\left[0, y_{0}\right] ; \\
& \\
& \quad f_{y y}(0,0)-\left[2 a_{y}^{1,1}(0,0)+a^{1,0}(0,0)\right] F_{2}^{\prime \prime}(0)-a^{1,1}(0,0) F_{2}^{\prime \prime \prime}(0) \\
& \quad-\left[2 a_{y}^{0,1}(0,0)+a^{0,0}(0,0)\right] F_{1}^{\prime \prime}(0)-a^{0,1}(0,0) F_{1}^{\prime \prime \prime}(0)=0 \\
&\left.\mathrm{~h}^{\prime}\right)-c b_{2} a^{1,1}(0, y)+b_{2} a^{1,0}(0, y)-c b_{1} a^{0,1}(0, y)+b_{1} a^{0,0}(0, y)=0,0 \leqslant y \leqslant y_{0} ; \\
&\left.\mathrm{j}^{\prime}\right) f(0, y)-a^{1,1}(0, y) F_{2}^{\prime}(y)-a^{1,0}(0, y) F_{2}(y)-a^{0,1}(0, y) F_{1}^{\prime}(y)-a^{0,0}(0, y) F_{1}(y)=0, \\
& 0 \leqslant y \leqslant y_{0} .
\end{aligned}
$$

ТЕОРема 6. Пусть выполнены условия (5), (36), (39) и (40). Тогда при выполнении а') задача (1)-(4) однозначно разрешима.

Если же имеет место $\left.\mathrm{b}^{\prime}\right)$, то при $\left.\mathrm{c}^{\prime}\right)$ задача (1)-(4) однозначно разрешима при дополнительном выполнении $\left.\mathrm{d}^{\prime}\right)$.

Далее, в случае, когда имеют место $\left.\mathrm{b}^{\prime}\right)$ u $\left.\mathrm{e}^{\prime}\right)$, при $\mathrm{f}^{\prime}$ ) задача (1)-(4) однозначно разрешима, если дополнительно выполнены $\left.\mathrm{d}^{\prime}\right)$ и $\mathrm{g}^{\prime}$ ).

Наконеи, пусть имеют место $\left.\left.\mathrm{b}^{\prime}\right), \mathrm{e}^{\prime}\right)$ u $\left.\mathrm{h}^{\prime}\right)$. Тогда для разрешимости задачи (1)-(4) необходимо и достаточно, чтобы выполнялось $\left.\mathrm{j}^{\prime}\right)$, причем соответствующая (1)-(4) однородная задача имеет бесконечное мноэсество линейно независимых решений, которые согласно (7) даются формулой

$$
\begin{aligned}
u_{0}(x, y)=- & \int_{0}^{y}\left[v(0, \eta ; x, y) \nu_{1}^{\prime}(\eta)+\left(a^{2,0} v\right)(0, \eta ; x, y) \nu_{1}(\eta)\right] d \eta-\int_{0}^{y}\left[v_{x y}(0, \eta ; x, y)\right. \\
& \left.-\left(a^{2,0} v\right)_{x}(0, \eta ; x, y)-\left(a^{1,1} v\right)_{y}(0, \eta ; x, y)+\left(a^{1,0} v\right)(0, \eta ; x, y)\right] \psi_{1}(\eta) d \eta \\
& -\left(a^{1,1} v\right)(0, y ; x, y) \psi_{1}(y)+v_{x}(0, y ; x, y) \psi_{1}(y), \quad(x, y) \in \bar{D}_{0},
\end{aligned}
$$


где $\psi_{1}$ и $\nu_{1}$ представимы по формулам (41), в которых $\psi_{*}$ - произвольная функиия

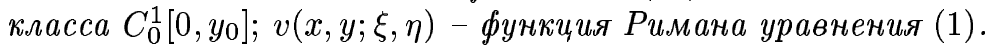

ЗАмЕЧАнИЕ 5. При вьполнении условий b), c), d) (соответственно $\left.\left.\left.\mathrm{b}^{\prime}\right), \mathrm{c}^{\prime}\right), \mathrm{d}^{\prime}\right)$ ) или b), e), f), d), g) (соответственно $\left.\left.\left.\left.\left.b^{\prime}\right), e^{\prime}\right), f^{\prime}\right), d^{\prime}\right), g^{\prime}\right)$ ) решение уравнения (33) (соответственно (42)) можно получить в явном виде.

ЗАмЕчАниЕ 6 . Аналогичным образом можно исследовать случаи, когда хотя бы одно из условий (39) или (40) нарушено.

ЗАмЕчАнИЕ 7. Коэффициенты и правые части граничных условий (3), (4) не присутствуют в явном виде в формулировании условий $\left.\left.\mathrm{a}^{\prime}\right)-\mathrm{j}^{\prime}\right)$, что было сделано с целью их компактной записи.

ЗАмЕЧАнИЕ 8. С помощью вьше приведенных способов аналогичным образом проводится исследование случая, когда условия (5) и (6) одновременно нарушаются всюду на отрезках $O P_{1}^{0}$ и $O P_{2}^{0}$ соответственно.

\section{СПИСОК ЦИТИРОВАННОЙ ЛИТЕРАТУРЫ}

[1] Хермандер Л. Линейные дифференциальные операторы с частными производными. М.: Мир, 1965.

[2] Бейли Н. Математика в биологии и медицине. М.: Мир, 1970.

[3] Васильев Ф. П. Методы решения экстремальных задач. М.: Наука, 1981.

[4] Виноградова М. Б., Руденко О. В., Сухоруков А. П. Теория волн. М.: Наука, 1979.

[5] Лаврентьев М. М., Романов В.Г., Васильев В. Г. Многомерные обратные задачи для дифференциальных уравнений. Новосибирск, 1969.

[6] Буллаф Р., Кодри Ф. Солитоны. М.: Мир, 1983.

[7] Чудновский А. Ф. Теплофизика почв. М.: Наука, 1976.

[8] Jokhadze O. On a Darboux problem for a third order hyperbolic equation with multiple characteristics // Georgian Math. J. 1995. V. 2. № 5. P. 469-490.

[9] Jokhadze O. General Darboux type problem for a third order equation with dominated lower terms // Bull. Acad. Sci. Georgia. 1996. V. 154. № 3. P. 344-347.

[10] Джохадзе О. М. Задача типа Дарбу для уравнения третьего порядка с доминирующими младшими членами // Дифференц. уравнения. 1996. Т. 32. № 4. С. 523-535.

[11] Jokhadze O. Boundary value problems in the plane for higher-order hyperbolic (pseudoparabolic) equations in angular and characteristic domains // Workshop in Partial Differential Equations. Germany: University of Potsdam, 1999. P. 17-18.

[12] Бицадзе А. В. Некоторые классы уравнений в частных производных. М.: Наука, 1981.

[13] Kharibegashvili S. Goursat and Darboux type problems for linear hyperbolic partial differential equations and systems // Memoirs Diff. Equations Math. Phys. Tbilisi. 1995. V. 4.

[14] Бицадзе А. В. Влияние младших членов на корректность постановки характеристических задач для гиперболических систем второго порядка // Докл. АН СССР. 1975. Т. 225. № 1. C. $31-34$.

[15] Солдатов А.П., Шхануков М.Х. Краевые задачи с общим нелокальньм условием А. А. Самарского для псевдопараболических уравнений высокогопорядка // Докл. АН СССР. 1987. T. 297. № 3. C. 547-552.

[16] Шхануков М. Х. О некоторых краевых задачах для уравнения третьего порядка, возникающих при моделировании фильтрации жидкости в пористых средах // Дифференц. уравнения. 1982. T. 18. № 4. C. 689-699. 